\title{
Sub-lethal pesticide exposure erases colour vision memory and affects flower constancy in foraging honey bees
}

Christopher Mayack ( $\nabla$ cmayack@sabanciuniv.edu )

Sabancı University https://orcid.org/0000-0003-0213-2149

\section{Tuğçe Rükün}

Sabanci University

\section{Neslim Ercan}

Sabanci University

\section{Ece Canko}

Sabanci University

\section{Bihter Avşar}

Sabanci University

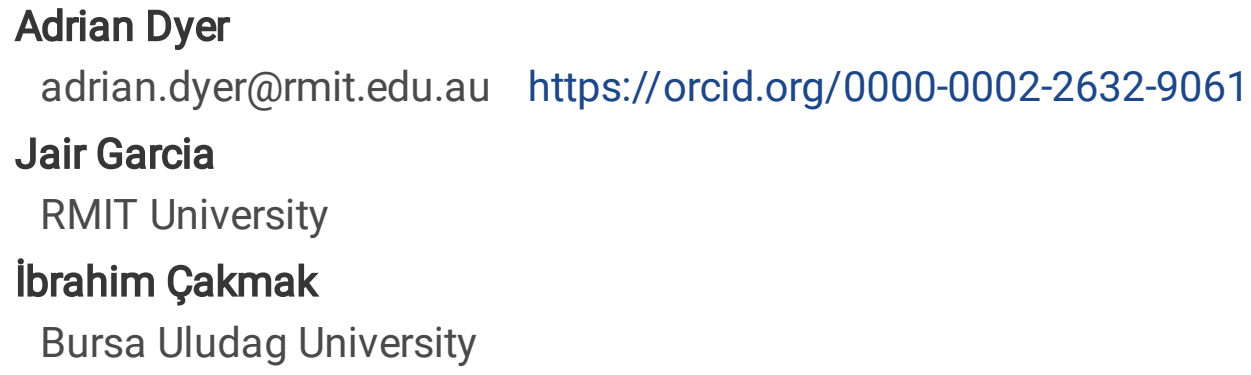

\section{Biological Sciences - Article}

Keywords: Imidacloprid, Colour vision, Apis mellifera, Opsin, CaMKII, CREB, Flower constancy

Posted Date: December 1st, 2021

DOI: https://doi.org/10.21203/rs.3.rs-1127589/v1

License: (c) (i) This work is licensed under a Creative Commons Attribution 4.0 International License. Read Full License 


\section{Abstract}

Neonicotinoid pesticide use has increased around the world despite accumulating evidence of their potential detrimental sub-lethal effects on the behaviour and physiology of bees, and its contribution to the global decline in bee health. Whilst flower colour is considered as one of the most important signals for foraging honey bees, the effects of pesticides on colour vision and memory retention remain unknown. We trained free flying foragers to an unscented artificial flower patch presenting yellow flower stimuli to investigate if sub-lethal levels of imidacloprid would disrupt the acquired association made between flower colour and food reward. We found that for concentrations higher than $4 \%$ of LD50 foraging honey bees no longer preferentially visited the yellow flowers and bees reverted back to baseline foraging preferences for blue flowers, with a complete loss of flower constancy. Higher pesticide dosages also resulted in a significant decrease in CaMKII and CREB gene expression, revealing a plausible mechanism to explain the disruption of bee foraging performance. Within important bee pollinators, colour vision is highly conserved and essential for efficient nutrition collection and survival. We thus show that to maintain efficient pollination services bees require environments free from neonicotinoid pesticides.

\section{Introduction}

Pollinators provide vital ecosystem services and serve as keystone species for the maintenance of biodiversity and agricultural production. Bees are one of the most abundant and important species for pollination services, which is worth around 235 - 577 billion USD ${ }^{1}$. Some crops are almost entirely dependent on bee pollination services, while for other self-pollinated crops the yield and quality of produce is significantly increased with bee pollination services ${ }^{2-4}$. Around the world most biotically pollinated flower colours show spectral characteristics of having evolved to suit the phylogenetically conserved colour vision of trichromatic bees ${ }^{5-8}$. Interestingly, bee species around the world appear to most frequently have a preference for short wavelength rich bluish colours ${ }^{9-12}$, which likely influenced flower colour evolution ${ }^{13}$. Bees in general play an important role in both pollination for ecology and also food production, and specifically the honeybee is a major model species for understanding potential stressors on essential pollination services. Ecological stressors such as reduction of foraging areas due to changes in land use, pathogens, pesticide exposure, and parasitic infections have been identified as probably causes for observed declines ${ }^{14-16}$. However, the effects of sub-lethal exposure to pesticides, on foraging behaviour of honey bees, in a field realistic setting, and the underlying neural mechanisms responsible for a potential disruption to vital sensory capabilities like colour vision learning and memory, remain unknown.

Pesticides and neonicotinoids in particular, have been identified as important agents responsible for bee declines due to their damaging effects on survival and reproduction, even at sub-lethal levels of exposure $^{17-19}$. Neonicotinoid pesticides have become the most widely used insecticides in the world since 1990s and are now found in the agricultural soil, pollen, and nectar of treated crops. Thus, forager bees 
are known to be easily exposed to sub-lethal levels of neonicotinoid pesticides, on a regular basis, from the surrounding environment ${ }^{19-21}$. Foraging honeybees are responsible for collecting all of the food for the bee hive which includes nectar and pollen, so they are crucial for the survival of the colony as a whole ${ }^{22}$. Recently, it has been shown that imidacloprid and thiamethoxam has a variety of sub-lethal effects on foraging bees floral odour learning and memory performance ${ }^{23,24}$ and navigational homing ability to return back to the hive ${ }^{25}$.

Flower colour is used not only to locate the flower, and where the nectar and pollen is located in the flower, but bees can learn colour and associate this with the food reward obtained from the flower via associative conditioning ${ }^{26,27}$, this signal is also used to remember this food source, which determines how likely a bee will return to this food source (flower constancy) or recruit other bees to this food source in the future ${ }^{28,29}$. Exposures to sub-lethal levels of pesticides may have a direct impact on not only the survival of the colony, but may decrease pollination efficiency services for a particular plant crop species due to the loss of flower constancy from poor associative learning stemming from altered colour vision perception and memory. Flower colours in addition to floral odours play a major role in the bee making an association between the flower and the reward it provides ${ }^{30}$ and it is considered as one of the most important signals for associative learning, which is necessary for foraging honey bees to reliably identify profitable flowers ${ }^{26,31}$, whilst avoiding alternative flower colours that offer no reward at that time $\mathrm{e}^{32,33}$.

Honeybees have trichromatic vision that contains three spectrally different photoreceptors including maximum sensitivity to UV $(344 \mathrm{~nm})$, blue $(436 \mathrm{~nm})$ and green $(544 \mathrm{~nm})$ regions of the spectrum, respectively ${ }^{34}$, and attentional mechanisms involving both learning and memory are essential to how bees can utilise colour information to efficiently collect nutrition ${ }^{26}$. Colour vision is enabled via opponent processes involving all three types of photoreceptors in sequential regions of the bee brain to generate a perceptual experience, whilst a separate visual pathway utilizes only input from the green sensitive receptor to enable fast achromatic vision, which is important for collision avoidance and target detection at a distance $26,27,35,36$.

We investigated if sub-lethal oral exposure of the neonicotinoid imidacloprid on free flying forager honey bees will alter floral visitation preferences based on colour and assessed the underlying mechanisms involved in colour vision sensory perception and memory. We show that at higher pesticide exposure level, preference and memory for the flower colour that the bee was trained to was disrupted, and instead bees reverted to baseline foraging colour preferences known to be present across bee species.

\section{Results}

\subsection{Imidacloprid effects on foraging behaviour}

A total of 160 honey bees were used in the experiment, out of these 116 honey bees $(72.5 \%)$ returned to the artificial flower board for the test phase of the experiment. The number of bees that returned to the artificial flower patch in the $4 \%$ and $10 \%$ treatment groups were significantly lower than expected (Chi- 
square goodness of fit: $\left.\chi^{2}=6.75, d f=1, P=0.009 ; \chi^{2}=4.17, d f=1, P=0.041\right)$. For the rest of the treatment groups 0,20 , and $40 \%$ there was no significant difference between the observed and expected number of returning forager bees to the artificial flower patch (Chi-square goodness of fit: $\chi^{2}=1.21, \mathrm{df}=$ $1, P=0.73, \chi^{2}=1.81, d f=1, P=0.18, \chi^{2}=1.57, d f=1, P=0.21$ ) (Fig. S3).

Overall imidacloprid had a significant effect on foraging behaviour of honey bees (Generalized Linear Model: $\left.\chi_{4,704}^{2}=20.28, P<0.001\right)$ and the preference of flower colour was dependent upon pesticide exposure (Generalized Linear Model: Colour ${ }^{\star}$ Pesticide Exposure Dose: $\chi_{4,704}^{2}=467.83, P<0.0001$ ). Honey bees in the control group and ones treated with $4 \%$ of the $L D_{50}$ value exhibited non-random choices (Generalized Linear Model: Parameter Estimate: Colour*Pesticide Exposure Dose 0\%: Colour^Pesticide Exposure Dose $\left.4 \%: \chi^{2}{ }_{1,704}=176.08, P<0.0001 ; \chi^{2}{ }_{1,704}=174.31, P<0.0001\right)$ as they had a high preference for yellow or similar wavelength reflecting hues to which they had been conditioned (red, green and yellow) (Chi-square goodness of fit: $\chi^{2}{ }_{1,60}=410.61, P<0.0001$ ). The $10 \%, 20 \%$, and $40 \%$ of the $L_{50}$ value treatments however demonstrated a significant preference for the blue like flower colours (blue, violet and white) instead of the yellow-like flowers found with the untreated and $4 \%$ of the $\mathrm{LD}_{50}$ value exposure groups (Chi-square goodness of fit: $\chi^{2}{ }_{1,54}=216.23, \mathrm{P}<0.0001$ ) (Fig. 2 ). Thus, in the higher dosage test groups the conditioning experience to the yellow stimulus had been extinguished.

\subsection{Colour vision}

Based on the absorbance values of the artificial flower colours used from the spectral analysis (Fig. 3A) and according to the model predicting honey bee colour vision perception, the highest to the lowest colour contrast versus the background of the artificial flower patch are as follows based on absolute conditioning: white > yellow > blue > green > purple > and red (Table S2). The colour contrast values among the artificial flowers indicate that white and blue along with white and green flowers are unlikely to be discriminated from each other by a bee considering absolute conditioning, while the yellow flower colour should be the easiest to discriminate from the rest of the flower colours (Table S3; Fig. 3B).

\subsection{Differential gene expression analysis}

There was a significant increase in relative gene expression of Blop in the forger bees exposed to $4 \%$ of the $L_{50}$ value (General linear mixed model: $F_{4,135}=2.83, P=0.027$ ), while for UVop and Lop 1 there was a significant increase in opsin relative gene expression for all of the pesticide doses $(4 \%, 10 \%, 20 \%$ and $40 \%$ of the $L_{50}$ value) $\left(F_{4,144}=4.21, P=0.0030 ; F_{4,144}=5.84, P<0.001 ;\right.$ Fig. $\left.4 A-C\right)$. FEN/ demonstrated only a significant difference between the 4 and $10 \%$ exposure treatment of the $L D 50$ value $\left(F_{4,92}=3.07, P\right.$ $=0.02$ ), while $C A M K I I$ and $C R E B$ had significantly consistent lower relative gene expression at the $10 \%$, $20 \%$, and $40 \%$ of the $L D_{50}$ exposure levels in comparison to the control bees $\left(F_{4,93}=15.81, P<0.001 ; F_{4,93}\right.$ $=9.34$, $P<0.001$; Fig. 4D-F).

\section{Discussion}


Foraging bees in the control group trained with absolute conditioning to associate yellow artificial flowers with a sucrose reward were subsequently able to recall the experience and significantly prefer choosing yellow and other similar long wavelength rich stimuli when presented in a mixed array of colours. A consistent finding was observed for bees experiencing $4 \%$ pesticide exposure. However, the foraging bees that received either 10,20 , or $40 \%$ pesticide exposure levels demonstrated a significant preference for blue-like flowers, consistent with innate foraging preferences of bees ${ }^{9}$. Thus, bees in these higher dosage experimental groups failed to either learn the association between the sucrose food reward and the yellow stimulus, or failed to enable the formation of memory traces required to recall rewarding colour information. The change in behaviour and gene expression occurred only after 30 minutes from being exposed to the pesticide orally, and according to our colour vision modelling, discriminating the yellow flower colour in comparison to the rest of the flower colours in the artificial flower patch should have been an easy cognitive task for the foraging honey bee. Therefore, it appears the acquired appetitive association of the yellow flower colour as evidenced in control and low dosage groups was completely absent at the higher pesticide exposure levels owing to the blocking of higher-level colour processing and/or memory formation of forager bees.

The change in foraging behaviour corresponded with a decrease in CAMKII and CREB gene expression at the higher 10, 20, and $40 \%$ exposure. CaMKII is important for long-term potentiation (LTP), a cellular mechanism of learning and memory. Phosphorylation of CaMKII results in the activation of cyclic AMP response element-binding protein (CREB), which then modulates transcription for the long-term memory of newly formed associations ${ }^{37,38}$, so our results suggest that mid and long-term memory is being affected as observed with the CaMKII and CREB gene expression and behavioural data. FEN-1 on the other hand did not show decreased gene expression in response to higher doses of pesticide exposure, however this is expected, because it is specifically associated with aversive learning ${ }^{39}$ and here the bees underwent appetitive positive reinforcement learning instead.

All three opsins demonstrated significantly higher gene expression at $4 \%$ exposure and at 10,20 , and $40 \%$ exposure there was an increase in UVop and Lop1 gene expression, but the differences observed are not substantial enough for altered colour vision perception due to the neural wiring and adaptation processes in the bee brain. Spectral signals are captured by the three spectrally different photoreceptors (UV, BLUE and Green-sensitive) in the bee eye and this information is projected along distinct visual pathways to sequential specialised regions in the brain including the lamina, medulla and lobula, before integration in higher levels including the central complex and mushroom bodies. To enable colour perception, opponent mechanisms are required and these are first evidenced the medulla and lobula levels of processing ${ }^{26}$. It is known that even considering large changes in relative stimulus colour such as when light colour varies that bee colour vision is very robust for making corrections via adaptation processes in early stages of visual processing ${ }^{40}$, and thus neural mechanisms can easily cope with even large fluctuations of the input signals. Our behavioural observations support this notion, at the $4 \%$ exposure level there was still a preference for the yellow flower. In addition, the foraging bees could still locate flowers at the higher exposure levels and preferred baseline floral preferences, further evidencing that colour was still 
functional and thus the loss of flower constancy from pesticide exposure was due to an interruption in associative learning.

We conclude that this disruption by higher dosage treatments is likely to be due to interference of midterm memory required to recall which flower colour was previously rewarding. These neurological effects are consistent with the mode of action of imidacloprid, which works by blocking the acetylcholine receptors, which triggers the depolarization of the postsynaptic membranes in the central nervous system. Therefore, it acts as nicotinic acetylcholine receptor agonist (nAChR) and competes for the target site of acetylcholine. Acetylcholine activity is present in the inner chiasm between the medulla and lobula of the brain as well as the central nervous system of insects ${ }^{41}$. The retinotopic organization is reversed again in the inner chiasm and chromatic properties of neurons are amplified in the lobula that contain distinct colour-opponent neurons and it is suggested that the visual neuropils rely on cholinergic signal transmission in bees ${ }^{42}$. Therefore, it is plausible that neonicotinoids mode of action can affect vision processing in the bee brain as there is a spatial overlap where vision is processed and cholinergic signal transmission is involved.

We propose that there is likely to be a threshold value in between the 4 and $10 \%$ exposure levels for imidacloprid to produce these physiological and behavioural effects, which was lower than expected and is within the range of realistic exposure levels from the agricultural environment ${ }^{43}$. At the $10 \%, 20 \%$, and $40 \% \mathrm{LD}_{50}$ doses there was not a significantly lower proportion of bees returning back to the bee board. Therefore, the loss of flower constancy for the yellow-like flowers in this study cannot be attributed to only testing a subset of foragers being able to return back to the artificial flower patch over time.

In summary, we show that sub-lethal imidacloprid pesticide exposure extinguishes the ability for the honey bee to return back to the flower that it was conditioned on based on colour cues. The cause is likely to be from the loss of colour vision memory formation as indicated by the changes in gene expression profiles in the honey bee brain. Bee pollinators that have a high level of flower constancy are critical for a successful pollination event because they are likely to visit flowers of the same species resulting in a higher probability of a successful plant reproductive event. Consequently, without flower constancy there will be an alteration of plant-pollinator co-evolution trajectories, the destabilization of plant-pollinator networks, loss of plant biodiversity, agriculture productivity, and ecosystem stability around the world ${ }^{44}$. As the debate of neonicotinoid use persists due to its potential harmful effects on bees, here we show a dramatic effect on colour vision which is central to foraging success, pollination services for crop production and biodiversity, as well as the survival of the honey bee.

\section{Declarations}

\section{Data Accessibility Statement}

All raw data has been made available to the public and can be found using the following link https://osf.io/bepq5/. 


\section{Acknowledgements}

We would like to thank Dr. Cleva W. Ow-Yang for her assistance in obtaining the artificial flower reflectance values using the UV-VIS-NIR integrating spectrophotometer.

\section{Funding}

This work was supported by a TUBITAK 3501 grant: $118 Z 157$ to CM.

\section{Ethics}

No special ethical approval is required to carry out this research.

\section{Competing interests}

The authors declare that there are no competing interests.

\section{Authors Contributions}

The original conceptualization of the study was performed by $\mathrm{CM}, \mathrm{TR}, \mathrm{NE}$, IÇ, and EC. CM, BA, TR, NE, and EC were responsible for carrying out the study and collecting the data. CM, BA, JG, and AGD conducted the data analysis. CM, IÇ, JG, and AGD provided the supervision for the study, the research facilities, and software. Funding for the study was obtained by CM. CM, AGD and TR were primarily responsible for writing the manuscript. All authors contributed to reviewing and editing the manuscript.

\section{References}

1 Potts, S. G. et al. Safeguarding pollinators and their values to human well-being. Nature 540, 220229, doi:10.1038/nature20588 (2016).

2 Klein, A. et al. Importance of pollinators in changing lanscapes for world crops. Proceedings of the Royal Society London B:Biological Sciences 274, 303-313 (2007).

3 Oz, M., Karasu, A., Cakmak, I., Goksoy, A. \& Ozmen, N. Effect of honeybees pollination on seed setting, yield and quality characteristics of rapeseed (Brassica napus oleifera). Indian Journal of Agricultural Sciences 78, 680-683 (2008). 
4 Oz, M., Karasu, A., Cakmak, I., Goksoy, A. \& Metin, Z. Effects of honeybee (Apis mellifera) pollination on seed set in hybrid sunflower (Helianthus annuus L.). African Journal of Biotechnology 8 (2009).

5 Chittka, L. \& Menzel, R. The evolutionary adaptation of flower colours and the insect pollinators' colour vision. Journal of Comparative Physiology A 171, 171-181, doi:10.1007/BF00188925 (1992).

6 Dyer, A. G. et al. Parallel evolution of angiosperm colour signals: common evolutionary pressures linked to hymenopteran vision. Proceedings of the Royal Society B: Biological Sciences 279, 3606-3615, doi:doi:10.1098/rspb.2012.0827 (2012).

7 Dyer, A. G. et al. Fragmentary blue: resolving the rarity paradox in flower colors. Frontiers in Plant Science 11, doi:10.3389/fpls.2020.618203 (2021).

8 Dalrymple, R. L. et al. Macroecological patterns in flower colour are shaped by both biotic and abiotic factors. New Phytologist 228, 1972-1985, doi:https://doi.org/10.1111/nph.16737 (2020).

9 Giurfa, M., Núñez, J., Chittka, L. \& Menzel, R. Colour preferences of flower-naive honeybees. Journal of Comparative Physiology A 177, 247-259, doi:10.1007/BF00192415 (1995).

10 Morawetz, L., Svoboda, A., Spaethe, J. \& Dyer, A. G. Blue colour preference in honeybees distracts visual attention for learning closed shapes. Journal of Comparative Physiology A 199, 817-827, doi:10.1007/s00359-013-0843-5 (2013).

11 Raine, N. E. \& Chittka, L. The Adaptive Significance of Sensory Bias in a Foraging Context: Floral Colour Preferences in the Bumblebee Bombus terrestris. PLoS ONE e556, 1-8 (2007).

12 Dyer, A. G. et al. Innate colour preferences of the Australian native stingless bee Tetragonula carbonaria Sm. Journal of comparative physiology. A, Neuroethology, sensory, neural, and behavioral physiology 202, 603-613, doi:10.1007/s00359-016-1101-4 (2016).

13 Dyer, A. G. et al. Fragmentary blue: resolving the rarity paradox in flower colors. Front Plant Sci 11, 618203, doi:10.3389/fpls.2020.618203 (2020).

14 Goulson, D., Nicholls, E., Botias, C. \& Rotheray, E. L. Bee declines driven by combined stress from parasites, pesticides, and lack of flowers. Science 347, 10, doi:10.1126/science.1255957 (2015).

15 Mayack, C. et al. Environmental exposures associated with honey bee health. Chemosphere, 131948, doi:https://doi.org/10.1016/j.chemosphere.2021.131948 (2022).

16 Naug, D. Nutritional stress due to habitat loss may explain recent honeybee colony collapses. Biological Conservation 142, 2369-2372, doi:10.1016/j.biocon.2009.04.007 (2009). 
17 Siviter, H. et al. Agrochemicals interact synergistically to increase bee mortality. Nature 596, 389392, doi:10.1038/s41586-021-03787-7 (2021).

18 Mayack, C. \& Boff, S. LD50 Values may be miseleading predictors of neonicotimoid toxicity across different bee species. Uludağ Arıcılık Dergisi 19, 19-33, doi:DOI: 10.31467/uluaricilik.568251 (2019).

19 Rundlöf, M. et al. Seed coating with a neonicotinoid insecticide negatively affects wild bees. Nature 521, 77-U162 (2015).

20 Wood, T. J. \& Goulson, D. The environmental risks of neonicotinoid pesticides: a review of the evidence post 2013. Environ. Sci. Pollut. Res. 24, 17285-17325, doi:10.1007/s11356-017-9240-x (2017).

21 Tsvetkov, N. et al. Chronic exposure to neonicotinoids reduces honey bee health near corn crops. Science 356, 1395-1397, doi:10.1126/science.aam7470 (2017).

22 Winston, M. L. The biology of the honey bee. (Harvard University Press, 1987).

23 Çakmak, i. et al. Effects of thiamethoxam on the behavior of foraging honey bees with artificial flower. Uludag Aricilik Dergisi 18, 2-13 (2018).

24 Decourtye, A. et al. Imidacloprid impairs memory and brain metabolism in the honeybee (Apis mellifera L.). Pesticide Biochemistry and Physiology 78, 83-92, doi:10.1016/j.pestbp.2003.10.001 (2004).

25 Fischer, J. et al. Neonicotinoids Interfere with Specific Components of Navigation in Honeybees. PLOS ONE 9, e91364 (2014).

26 Dyer, A. G., Paulk, A. C. \& Reser, D. H. Colour processing in complex environments: insights from the visual system of bees. Proceedings of the Royal Society B: Biological Sciences 278, 952-959, doi:10.1098/rspb.2010.2412 (2011).

27 Hempel de Ibarra, N., Vorobyev, M. \& Menzel, R. Mechanisms, functions and ecology of colour vision in the honeybee. Journal of Comparative Physiology A 200, 411-433, doi:10.1007/s00359-0140915-1 (2014).

28 Wells, H. \& Sanderson, C. The flower fidelity of the honey bee. Uludağ Arıcılık Dergisi 5, 32-41, doi:https://dergipark.org.tr/en/pub/uluaricilik/issue/53635/162583 (2005).

29 Chittka, L., Thomson, J. D. \& Waser, N. M. Flower constancy, insect psychology, and plant evolution. Naturwissenschaften 86, 361-377, doi:10.1007/s001140050636 (1999).

30 Kantsa, A. et al. Community-wide integration of floral colour and scent in a Mediterranean scrubland. Nature Ecology \& Evolution 1, 1502-1510, doi:10.1038/s41559-017-0298-0 (2017).

31 Dyer, A. G. \& Garcia, J. E. Color Difference and Memory Recall in Free-Flying Honeybees: Forget the Hard Problem. Insects 5, 629-638 (2014). 
32 Garcia, J. E., Phillips, R. D., Peter, C. I. \& Dyer, A. G. Changing How Biologists View Flowers-Color as a Perception Not a Trait. Frontiers in Plant Science 11, doi:10.3389/fpls.2020.601700 (2020).

33 Garcia, J. E., Shrestha, M. \& Dyer, A. G. Flower signal variability overwhelms receptor-noise and requires plastic color learning in bees. Behavioral Ecology 29, 1286-1297, doi:10.1093/beheco/ary127 (2018).

34 Avarguès-Weber, A., Mota, T. \& Giurfa, M. New vistas on honey bee vision. Apidologie 43, 244-268, doi:10.1007/s13592-012-0124-2 (2012).

35 Skorupski, P. \& Chittka, L. Differences in Photoreceptor Processing Speed for Chromatic and Achromatic Vision in the Bumblebee, Bombus terrestris. The Journal of Neuroscience 30, 3896-3903, doi:10.1523/jneurosci.5700-09.2010 (2010).

$36 \mathrm{Ng}$, L., Garcia, J. E. \& Dyer, A. G. Why colour is complex: Evidence that bees perceive neither brightness nor green contrast in colour signal processing. FACETS 3, 800-817, doi:10.1139/facets-20170116 (2018).

37 Eisenhardt, D. et al. The AmCREB gene is an ortholog of the mammalian CREB/CREM family of transcription factors and encodes several splice variants in the honeybee brain. Insect Mol Biol 12, 373382, doi:10.1046/j.1365-2583.2003.00421.x (2003).

38 Kamikouchi, A., Takeuchi, H., Sawata, M., Natori, S. \& Kubo, T. Concentrated expression of Ca2+/ calmodulin-dependent protein kinase II and protein kinase $\mathrm{C}$ in the mushroom bodies of the brain of the honeybee Apis mellifera L. The Journal of comparative neurology 417, 501-510, doi:10.1002/(sici)10969861(20000221)417:4<501::aid-cne8>3.0.co;2-4 (2000).

39 Saavedra-Rodríguez, L. et al. Identification of flap structure-specific endonuclease 1 as a factor involved in long-term memory formation of aversive Learning. The Journal of Neuroscience 29, 57265737, doi:10.1523/jneurosci.4033-08.2009 (2009).

40 Chittka, L., Faruq, S., Skorupski, P. \& Werner, A. Colour constancy in insects. Journal of comparative physiology. A, Neuroethology, sensory, neural, and behavioral physiology 200, 435-448, doi:10.1007/s00359-014-0897-z (2014).

41 Kreissl, S. \& Bicker, G. Histochemistry of acetylcholinesterase and immunocytochemistry of an acetylcholine receptor-like antigen in the brain of the honeybee. The Journal of comparative neurology 286, 71-84, doi:10.1002/cne.902860105 (1989).

42 Grünewald, B. \& Siefert, P. Acetylcholine and its receptors in honeybees: involvement in development and impairments by neonicotinoids. Insects 10, 420, doi:10.3390/insects10120420 (2019).

43 Stewart, S. D. et al. Potential exposure of pollinators to neonicotinoid insecticides from the use of insecticide seed treatments in the mid-southern United States. Environmental Science \& Technology 48, 
44 Wei, N. et al. Pollinators contribute to the maintenance of flowering plant diversity. Nature 597, 688-692, doi:10.1038/s41586-021-03890-9 (2021).

45 Karahan, A., Cakmak, I., Hranitz, J. M., Karaca, I. \& Wells, H. Sublethal imidacloprid effects on honey bee flower choices when foraging. Ecotoxicology 24, 2017-2025, doi:10.1007/s10646-015-1537-2 (2015).

46 Carreck, N. \& Ratnieks, F. The dose makes the poison: Have "field realistic" rates of exposure of bees to neonicotinoid insecticides been overestimated in laboratory studies? Journal of Apicultural Research 53, 607-614, doi:10.3896/IBRA.1.53.5.08 (2014).

47 Lourenço, A. P., Mackert, A., Cristino, A. D. \& Simoes, Z. L. P. Validation of reference genes for gene expression studies in the honey bee, Apis mellifera, by quantitative real-time RT-PCR. Apidologie 39, 372U333, doi:10.1051/apido:2008015 (2008).

48 Team, R. D. C. R: A language and environment for statistical computing, <http://www.Rproject.org.> (2008).

\section{Methods}

\section{1 Experimental setup}

A foraging board representing an artificial flower patch used was adapted from Karahan et al. (2015) The foraging board presented six spectrally different colours (blue, green, yellow, white, red or purple appearance considering human vision; (Paint Land enamel paint, Istanbul, Turkey; Fig S1). Stimuli colour was quantified for trichromatic bee vision using standard techniques (see Bee colour vision perception modelling below). These artificial flowers were randomly arranged on $90 \mathrm{~mm}$ long, $5 \mathrm{~mm}$ diameter dowels presenting respective stimuli against an achromatic grey background. Artificial flowers were made from $28 \mathrm{~mm} \times 28 \mathrm{~mm}$ Plexiglass squares with $6 \mathrm{~mm}$ thickness. At the centre of each square, there was a $5 \mathrm{~mm}$ diameter hole drilled, with a $5 \mathrm{~mm}$ depth in which a hidden nectar reward could be placed during the experiment.

\subsection{Bee colour vision perception modelling}

A UV-VIS-NIR integrating spectrophotometer, sphere attachment model UV-3100 (SHIMADZU Corporation, Kyoto, Japan) with a Halogen (RJ-5012) and Deuterium lamp (L6380) light source, was used to measure the reflectance spectra between $300 \mathrm{~nm}$ and $700 \mathrm{~nm}$ of the enamel paints used to produce the artificial flowers. Barium sulphate ( $100 \%$ reflectance) and black velvet ( $0 \%$ reflectance) controls were used to calibrate the instrument and data was managed by means of the UVProbe version 2.21 software (Shimadzu, Kyoto, Japan). For details please see the supplemental information. 


\subsection{Training phase}

Free flying forager honey bees, Apis mellifera anatoliaca, from three different colonies, were trained to an artificial flower patch using artificial yellow flower stimuli with non-scented $30 \%$ sucrose solution, $50 \mathrm{~m}$ away from the hive, which consisted of an artificial flower patch of 36 flowers (on a square achromatic wooden bee board) (Fig. S2). The yellow feeder stimuli were initially placed right in front of the hive to prime bees, and then moved incrementally to a distance $50 \mathrm{~m}$ away from the hive once active recruitment was enabled. At this point, the artificial feeder was then replaced with an artificial flower patch filled with each flower containing a reward of $4 \mu \mathrm{L}$ unscented $15 \%$ sucrose solution. Later in the season $30 \%$ sucrose solution was used in each flower stimuli to increase the motivation of the forager bees to return back to the artificial flower patch after pesticide treatment. In order to distinguish the bees being trained from the same hive we individually paint-marked bees either on the abdomen, thorax or both, as soon as they initially visited the feeder. After about $15-20$ bees were marked, any unmarked bees observed at the artificial feeder were removed using a glass vial and were released at the end of the experiment to avoid unnecessary competition from other bee hives.

\subsection{Experimental test phase}

The experiment took place between July 22, 2019 and August 30, 2019. Each trial contained 3 phases that proceeded sequentially: (1) Crop Attachment Phase, (2) Pesticide Exposure, and (3) Test Phase (Fig. 1). In the crop attachment phase each artificial flower on the bee board contained $4 \mu \mathrm{L}$ of $30 \%$ sucrose solution. After a bee consumed the reward, the flower was refilled manually using a micropipetter. After $30 \mathrm{~min}$, the bees were captured on the first flower that they landed on, before they could consume the food reward and were put in a $20 \mathrm{~mL}$ glass vial for pesticide exposure.

The pesticide exposure phase lasted $30 \mathrm{~min}$ and there were two parts: in the first part, bees were hand fed $5 \mu \mathrm{l}$ of unscented $30 \%$ sucrose solution, with a micropipetter and it was ensured that bee consumed all of the sucrose solution, mixed with nothing (control), or imidacloprid (PESTANAL ${ }^{\circledR}$, Sigma Aldrich) at the following doses: $0.00 \mathrm{ng} /$ bee, the negative control $(\mathrm{N}=31), 0.144 \mathrm{ng} /$ bee which is $4 \%$ of the $\mathrm{LD}_{50}(\mathrm{~N}=$ 30), $0.36 \mathrm{ng} /$ bee which is $10 \%$ of the $\operatorname{LD}_{50}(\mathrm{~N}=18), 0.72 \mathrm{ng} /$ bee which is $20 \%$ of the $\operatorname{LD}_{50}(\mathrm{~N}=20)$, and $1.44 \mathrm{ng} / \mathrm{bee}$ which is $40 \%$ of the $\mathrm{LD}_{50}(\mathrm{~N}=17)^{30}$. For each dose, $4-7$ trials were conducted and each time with a new set of bees. Each trial started with roughly 6 to 8 bees at a time. In the second part, after 15 min, captured bees were released and the flower patch remained in place without any food rewards for 15 min. This additional wait period ensured the oral ingestion of the pesticide. The sub-lethal doses and administration of neonicotinoid pesticides were adjusted according to previous studies for agricultural settings and the effects of pesticides for foraging bees, which conform to current national guidelines $\left(\right.$ e.g. $\left.{ }^{46}\right)$. 
During the test phase all artificial flowers were filled with $4 \mu \mathrm{L}$ of unscented $30 \%$ sucrose solution. The flowers were once again re-filled manually using a micropipetter after a bee consumed a reward during the $45 \mathrm{~min}$ test period. After each flower visitation, the colour of the flower visited was recorded. A visitation was defined only if the bee depleted the food reward that was placed at the centre of the flower. The pesticide treatment dose was blind to the observer and administered in random order. In between trials, flowers were washed with ethanol, rinsed with water, and air-dried to prevent any potential odour bias from the visitations of the bees to the artificial flowers.

\subsection{Colour vision gene expression assay}

Returning forager bees were collected using $20 \mathrm{~mL}$ glass vials and were placed on ice to be immobilized, these individuals were brought back to the lab. While in the vial, they were hand fed using a micropipettor with $5 \mu \mathrm{L}$ of unscented $30 \%$ sucrose solution (control) or mixed with the pesticide treatment of $4,10,20$ or $40 \%$ of the imidacloprid $L_{50}$ value. Each bee was held in the vial for a total of $15 \mathrm{~min}$ and we once again insured that all of the food was consumed by the bee. Then the bees were held in the vials for an additional 15 min without getting any food reward to mirror the timeline of the above field experiment. After this, 6 bees from a treatment group were placed in a metal bee cage $(10 \mathrm{~cm} \times 10 \mathrm{~cm} \times 6 \mathrm{~cm})$ with a sugar water feeder of unscented $30 \%$ sucrose solution. A total of 5 bee cages were used and 5 trials were conducted for each treatment group, yielding a total sample size of 30 bees for each treatment group. Bee cages were left in the lab by the window at room temperature which was approximately $23^{\circ} \mathrm{C}$ for 45 min. Then, bees were snap frozen and kept at $-80^{\circ} \mathrm{C}$ until further gene expression analysis.

\subsubsection{RNA extraction and CDNA synthesis}

For total RNA extraction, the head of the each bee sample was separated on ice and they were transferred to a $2 \mathrm{~mL}$ microcentrifuge tube. To isolate the RNA we used the Ez-10 Spin Column Total RNA Mini-Prep kit (Bio Basic Company, Canada) and followed the manufacturer's instructions. During the isolation, total RNA was treated with DNase I (EurX Molecular Biology Products, Poland) for the removal of DNA contamination. The purified RNA was measured using Thermo Scientific NanoDrop 2000/2000c (Thermo Fisher Scientific, US) spectrophotometer and then the concentration was standardized. The RNA integrity was checked by conducting a $1 \%$ agarose gel electrophoresis to determine if the RNA bands were clearly visible, visualized using a GelDoc EZ instrument (Bio-Rad, Hercules, CA, USA). cDNA synthesis was performed using OneScript cDNA synthesis kit (Applied Biological Materials, Canada) according to the manufacturer's instructions. All CDNA was stored at $-20^{\circ} \mathrm{C}$ until the further use for quantitative real-time PCR (qPCR). 
The three opsin genes (Blop, UVop, and Lop1) and the three memory-related genes (FENI, CAMKII, and $C R E B)$ were analysed for all of the pesticide treatments and the corresponding primer sets can be found in Table S1. Ribosomal protein 49 (RP49) was used as a reference gene since it has been shown as a stable housekeeping gene in Apis mellifera ${ }^{47}$.

qPCR assays were carried out in a final volume of $10 \mu \mathrm{L}$ using a BrightGreen 2X qPCR MasterMix-No Dye kit (Applied Biological Materials, Canada), which is suitable for the LightCycler 480 Real-Time PCR system (Roche Life Sciences, Germany). PCR conditions were as follows: $10 \mathrm{~min}$ at $95^{\circ} \mathrm{C}$, followed by 40 cycles of $15 \mathrm{~s}$ at $95^{\circ} \mathrm{C}, 30 \mathrm{~s}$ at $60^{\circ} \mathrm{C}$ and $30 \mathrm{~s}$ at $72^{\circ} \mathrm{C}$ ending with a melt curve analysis to account for the primer specificity. Each sample was run in duplicate and if a run failed then it was re-run again.

\subsection{Statistical analysis}

All statistical analyses were performed using SPSS software version 25 (IBM, city location) and R statistical package ${ }^{48}$. A generalized linear model with Poisson distribution was used to analyse the number of flower visits across the visible light spectrum as a function of pesticide exposure. Therefore, the number of flower visits served as the dependent variable, while colour was a fixed factor with six levels and the amount of pesticide exposure was considered as a continuous covariate. The trial and colony were considered as a random effect in the model. A separate two by two chi-square goodness of fit test was carried out where the number of visits were compared for blue like flowers versus yellow like flowers and these preferences were pooled for the control and $4 \%$ treated bees versus the 10,20 , and $40 \%$ of the $L_{50}$ treated bees. The gene expression data was found to be normally distributed, so a general linear mixed model, with bee cage as a random effect, pesticide treatment dose as the independent variable, and relative gene expression as the dependent variable was followed by a Tukey-HSD post hoc test to analyse each of the six gene targets.

\section{Figures}




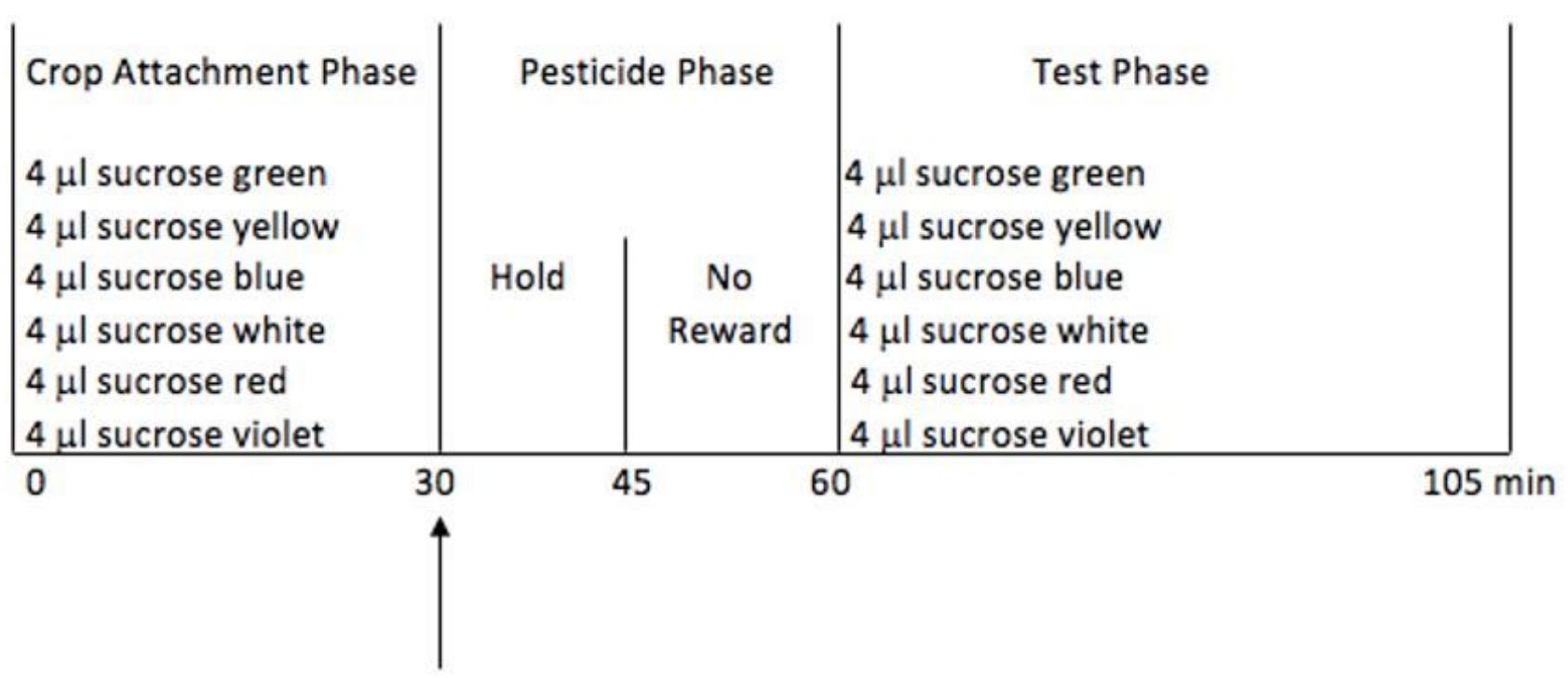

Pesticide Dose

\section{Figure 1}

A schematic diagram of the experimental design showed three phases of each trial that were run without a break. In the crop attachment phase, bees were trained to visit artificial flower patches. In the pesticide phase, imidacloprid doses were offered and allowed to digest by the bee. In the test phase, colour preferences of marked bees were recorded.

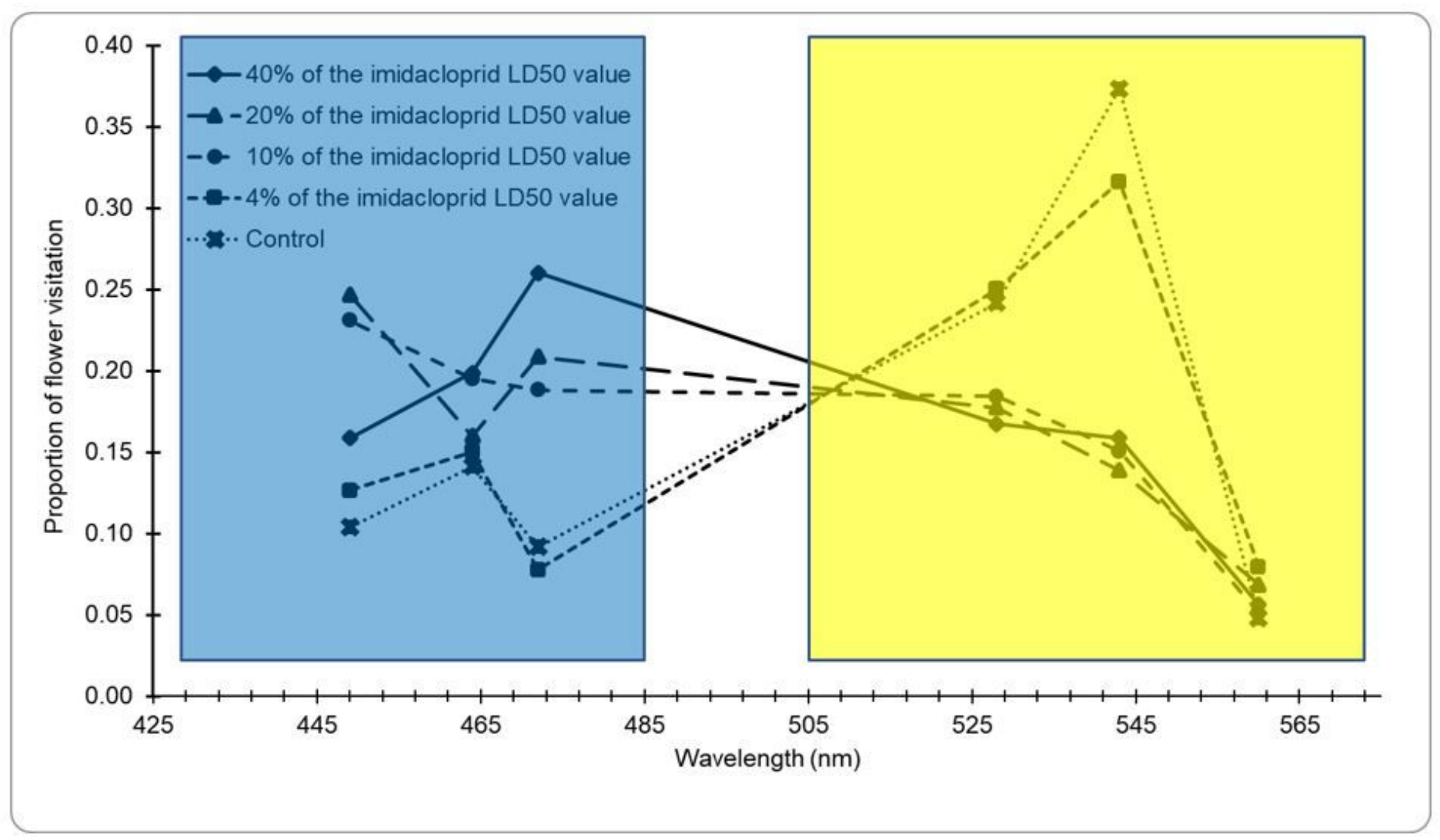


Figure 2

Proportion of flower visitation plotted as a function of flower wavelength. Forager bees were trained to an artificial flower patch and then they were orally exposed to imidacloprid (1.44 ng imidacloprid which equals $40 \%$ LD50, $0.36 \mathrm{ng}$ which equals $20 \%$ LD50, $0.72 \mathrm{ng}$ which equals $10 \%$ LD50, $0.144 \mathrm{ng}$ which equals $4 \%$ LD50, and $0 \mathrm{ng}$ which equals $0 \%$ LD50, the negative control). Afterwards, their visitations to the different coloured flowers, with the same exact food reward of $4 \mu \mathrm{l}$ of $30 \%$ sucrose solution, was recorded. The blue area represents where there is a short wavelength "blue like" flowers, while the yellowcoloured area represents the long wavelength "yellow like" flowers foraging bees can choose from.

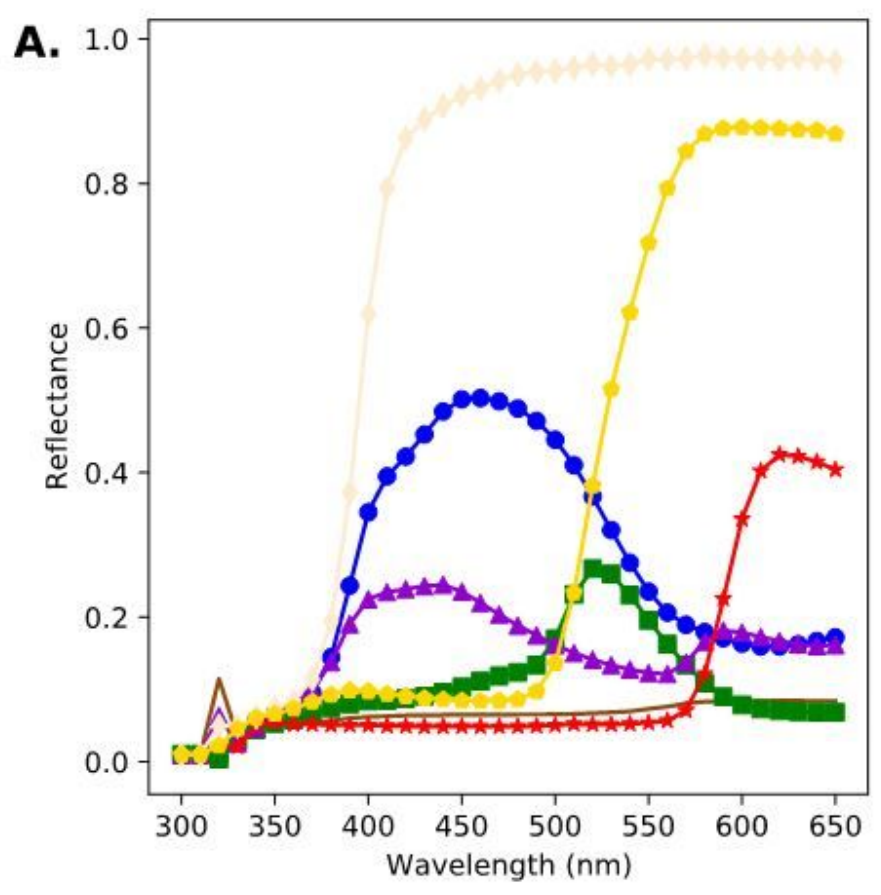

B.

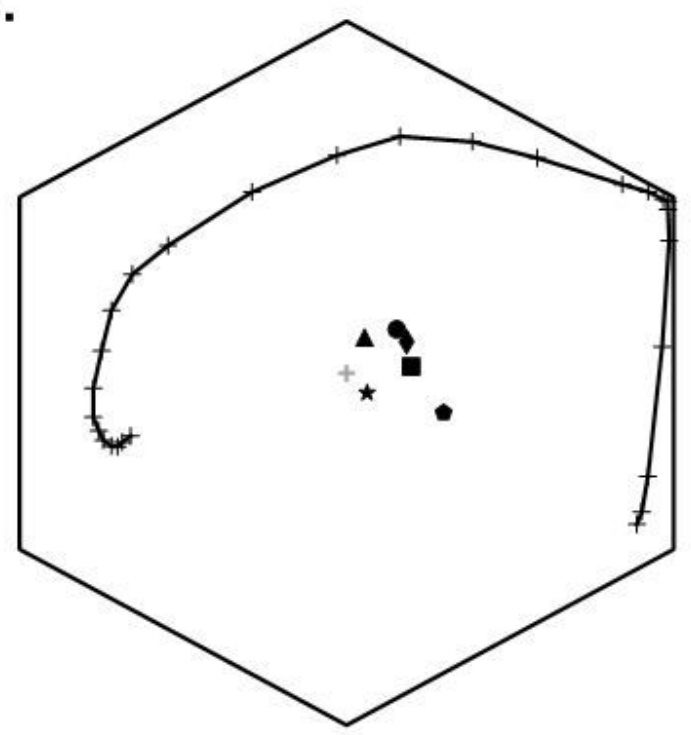

Figure 3

Spectral profiles of the colour stimuli used for the experiments (panel A.) and corresponding modelling in the hexagon space (panel B.). In panel A stimuli are represented by their chromatic appearance considering human colour vision: blue (circle marker), green (square marker), purple (triangle marker), red (star marker), white (diamond marker) and yellow (pentagram marker). Reflectance of the adapting background is represented by a brown line with no markers. Stimuli in the hex colour space (panel B) are represented by their respective markers in panel A. Spectral loci for A. mellifera in the hexagon space is indicated by the solid, black line with cross markers. 

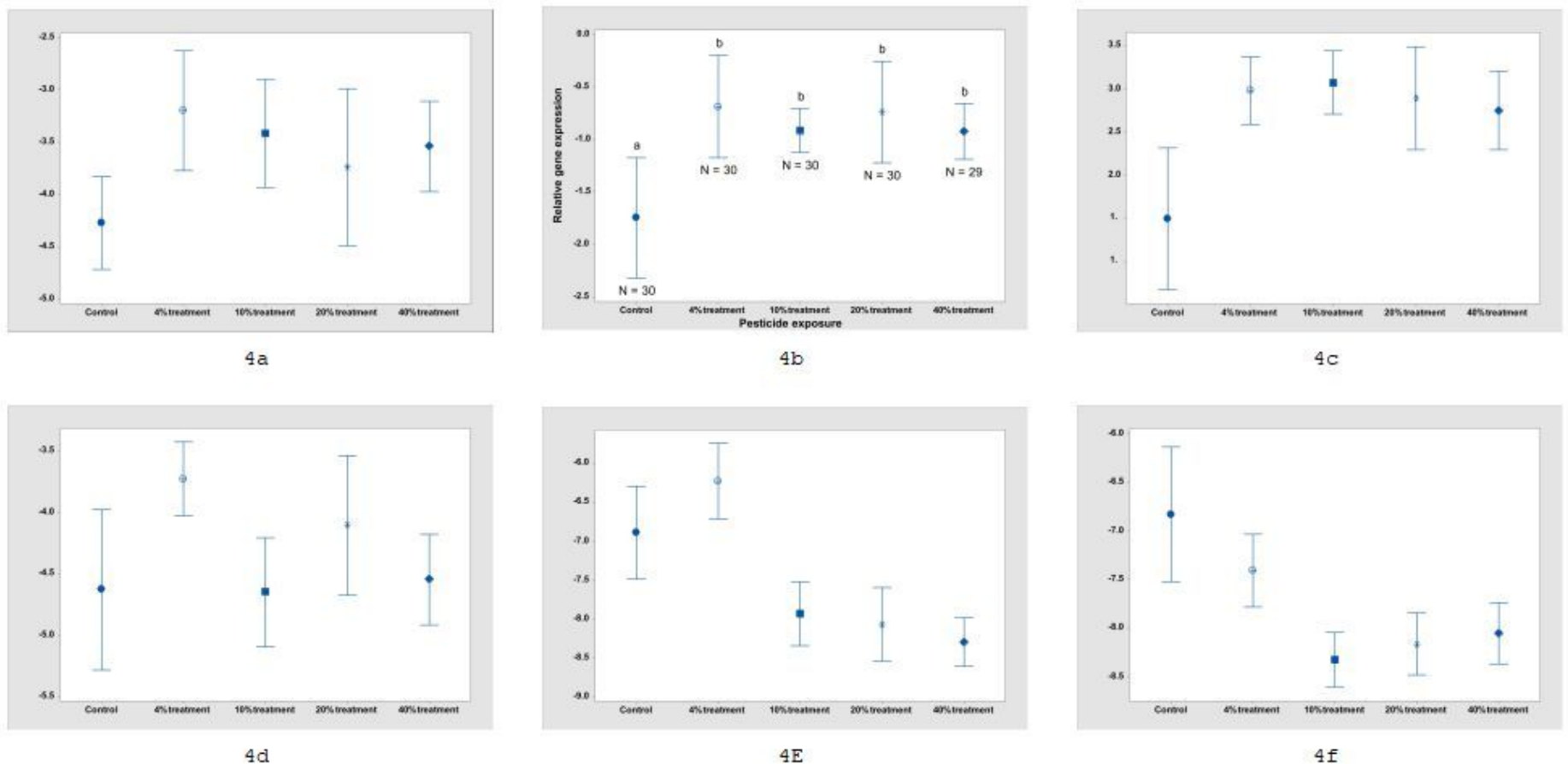

\section{Figure 4}

Relative gene expression of the three honey bee colour vision opsins (a) Blop, (b) UVop, and (c) Lop1 as well as the colour vision memory related neurological targets that include (d) FEN-1, (e) CaMKII, and (f) CREB. The individual dots, filled circle (control), open circle ( $4 \%$ of the LD50 value), filled square ( $10 \%$ of the LD50 value), asterisk ( $20 \%$ of the LD50 value) and filled diamond ( $40 \%$ of the LD50 value) represent the mean expression levels across the pesticide exposure treatments after being normalized using the $-\triangle \Delta$ CT method, while the error bars represent the $95 \%$ confidence intervals. The different letters above each bar indicate significant differences from a Tukey post hoc test. The numbers below each bar indicate the sample size for each group. 